\title{
MULTIOBJECTIVE AND MULTISCALE OPTIMIZATION OF COMPOSITE MATERIALS BY MEANS OF EVOLUTIONARY COMPUTATIONS
}

\author{
Witold Beluch, Adam DŁugosz \\ Silesian University of Technology, Institute of Computational Mechanics and Engineering, Gliwice, Poland \\ e-mail: witold.beluch@polsl.pl; adam.dlugosz@polsl.pl
}

\begin{abstract}
The paper deals with the multiobjective and multiscale optimization of heterogeneous structures by means of computational intelligence methods. The aim of the paper is to find optimal properties of composite structures in a macro scale modifying their microstructure. At least two contradictory optimization criteria are considered simultaneously. A numerical homogenization concept with a representative volume element is applied to obtain equivalent macro-scale elastic constants. An in-house multiobjective evolutionary algorithm MOOPTIM is applied to solve the considered optimization tasks. The finite element method is used to solve the boundary-value problem in both scales. A numerical example is attached.
\end{abstract}

Keywords: composite, numerical homogenization, multiobjective optimization, evolutionary algorithm

\section{Introduction}

Composites are structural materials which are increasingly used and constantly gain in popularity due to their properties. In particular, the favourable strength/weight ratio causes them to displace traditional structural materials such as metals and their alloys in many areas of technology. Their properties depend on such parameters as the properties of constituent materials, volume fraction of the constituents as well as shape and location of the reinforcement. Proper manipulation of such parameters allows obtaining the desired behaviour of composite structures.

In order to obtain the best (for given criteria) properties, optimization methods have to be applied. Since the application of conventional, typically gradient-based, optimization methods for composites may encounter difficulties due to multimodality and discontinuity of the objective function, it is reasonable to use global optimization methods, like bio-inspired optimization algorithms, e.g. evolutionary algorithms, artificial immune systems or particle swarm optimizers (Michalewicz and Fogel, 2004; De Castro and Timmis, 2002; Kennedy and Eberhart, 2001).

In real optimization problems, it is very often necessary to consider more than one criterion at the same time. If the criteria are contradictory, the optimization task belongs to multiobjective ones. The dedicated implementations of bio-inspired global optimization methods can be applied to solve multiobjective optimization tasks. A survey of the state of the art of the multiobjective evolutionary algorithms can be found in Zhoua et al. (2011).

Proper determination of the effect of micro-structure of heterogeneous materials on their behaviour at the macro level allows the optimal design of heterogeneous materials. Multiscale optimization allows designing materials in one scale level to obtain the desired properties of the material on different scale(s). Different homogenization methods are typically applied to perform calculations in more than one scale in a reasonable time (Kouznetsova, 2002; Buryachenko, 2007; Zohdi and Wriggers, 2005).

There are numerous approaches to the multiscale modelling. Analytical or semi-analytical methods are typically used to determine the equivalent material constants for inclusions or voids of regular shape, e.g. circular, elliptical or spherical (Eshelby, 1957; Bensoussan et al., 1978). 
The applied in the present paper attitude is based on numerical homogenization methods belonging to so-called upscaling methods. Simulation in this group of homogenization methods is carried out hierarchically in different scales utilizing the representative volume element attitude. The computational intelligence methods have been successfully applied by the authors to multiobjective optimization problems of composite structures at the macro scale only, see e.g. Beluch et al. (2008). The application of computational intelligence methods for the single-objective multiscale identification of material constants in heterogeneous materials was presented by Beluch and Burczyński (2014).

\section{Multiscale modelling}

Many structural materials like composites, porous materials or polycrystalline materials are nonhomogeneous on a certain observation level. In order to model such materials more precisely, considerations in a macro scale only may be insufficient. Taking into account different scales allows modelling different geometric and material properties of the structures. A macro-scale model may contain various types of external loads (mechanical, thermal, electrical, etc.). Meso and micro scales make it possible to consider such elements as discontinuities or imperfections, like cracks, voids, inclusions or surface roughness (Nemat-Nasser and Hori, 1993; Vernerey and Kabiri, 2014). A nano-scale level includes e.g. crystal lattice defects while an atom-scale level allows incorporating molecular mechanics effects (Burczyński et al., 2007). The number of considered scales depends on the required accuracy of the model (Ilic and Hackl, 2009).

The proper determination of the influence of the micro-structure of heterogeneous materials on their behaviour at the macro level allows optimal designing of them. An appropriate selection of the component materials, geometry and volume ratio of constituents allows creating materials with desired properties, including those which cannot be obtained with the application of homogeneous materials only (Takano and Zako, 2000).

The direct application of more than one scale in numerical calculations by means of numerical methods such as the finite element method (FEM) (Zienkiewicz and Taylor, 2000) or boundary element method (BEM) (Brebbia and Dominiguez, 1989) leads to systems with such large numbers of degrees of freedom that they are very hard or even impossible to be solved. In order to overcome this problem, different homogenization techniques are employed. In the present paper, numerical homogenization techniques are applied to find the parameters of the equivalent material for composite structures. The behaviour of heterogeneous media is described by differential equations with discontinuous coefficients like elastic constants in linear-elastic problems. The aim of the numerical homogenization is to determine continuous, effective coefficients of differential equations which are applied to a higher scale. A typical attitude in the numerical homogenization consists in the determination of constitutive relation between averaged field variables, like stresses and strains (Ptaszny and Fedeliński, 2011).

\subsection{Numerical homogenization of heterogeneous materials}

Numerical homogenization techniques belong to upscaling methods which perform hierarchical simulation in particular scales and make use of the representative volume element (RVE) concept (Hill, 1963). They allow obtaining macroscopically homogeneous, equivalent materials which behave in the macro scale as microscopically heterogeneous ones.

RVEs are used for globally or locally periodical structures. RVE represents the structure of the whole medium or its part, so it has to include all information required for a thorough description of the structure and properties of the medium (Hashin, 1964). 
Numerical homogenization can be performed under certain conditions:

a) The principle of the scales separation requires that RVE size $l_{R V E}$ must be significantly greater than the microstructure characteristic dimensions $l_{\text {micro }}$ and considerably smaller than the characteristic dimensions $l_{\text {macro }}$ in the macro scale (Zohdi and Wriggers, 2005)

$$
l_{\text {micro }} \ll l_{R V E} \ll l_{\text {macro }}
$$

It is commonly assumed that the RVE is the smallest possible volume representing the entire medium or its part. RVE should meet two conflicting criteria: be large enough to be representative of the entire structure and as small and uncomplicated (geometrically and materially) as possible in order to carry out its precise numerical analysis (Madi et al., 2006). In the case of fully regular structures (commonly used for fiber-reinforced composites), RVE may contain only one centrally placed core. Such an RVE is called a unit cell.

b) Averaging is performed according to the relation

$$
\langle\cdot\rangle=\frac{1}{|V|} \int_{V}(\cdot) d V
$$

where $\langle\cdot\rangle$ is the averaged value of the field under consideration, $V$ - RVE volume.

c) The condition specifying the equality of the average energy density in the micro scale and the macroscopic energy density at the point of macrostructure corresponding to the RVE (Hill condition) has the form (Kröner, 1972)

$$
\left\langle\sigma_{i j} \varepsilon_{i j}\right\rangle=\left\langle\sigma_{i j}\right\rangle\left\langle\varepsilon_{i j}\right\rangle
$$

where: $\sigma_{i j}$ and $\varepsilon_{i j}$ are stress and strain tensors in the micro scale.

d) Appropriate boundary conditions, e.g. periodic boundary conditions (Kouznetsova, 2002): periodic displacements and anti-periodic tractions on opposite faces of the RVE, as shown in Fig. 1, are

$$
\begin{array}{lll}
u_{i}^{+}=u_{i}^{-} & \forall r \in \partial V: & n_{i}^{+}=-n_{i}^{-} \\
t_{i}^{+}=-t_{i}^{-} & \forall r \in \partial V: & n_{i}^{+}=-n_{i}^{-}
\end{array}
$$

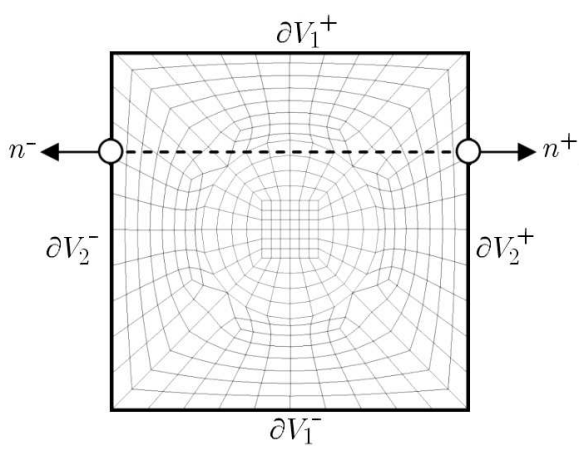

Fig. 1. RVE boundaries for periodic boundary conditions

In addition to the boundary conditions, strain boundary conditions from the higher scale are imposed on every RVE (localization). If FEM is applied to solve the boundary-value problem in both scales, the RVE is assigned to each integration point in the micro scale (Kuczma, 2014).

Averaged stresses, calculated according to Eq. (2.2), are obtained as a result of numerical computations in the micro scale (homogenization). Averaged stresses are transferred to the 
higher scale in order to calculate homogenized material parameter values at the macro scale taking into account the constitutive equation for the homogenized material. Assuming that the considered composites can be treated as orthotropic materials in the plane strain state, the constitutive equation in the Voight notation has the form (Gibson, 2012)

$$
\left[\begin{array}{l}
\left\langle\sigma_{11}\right\rangle \\
\left\langle\sigma_{22}\right\rangle \\
\left\langle\sigma_{12}\right\rangle
\end{array}\right]=\left[\begin{array}{ccc}
Q_{11} & Q_{12} & 0 \\
& Q_{22} & 0 \\
\cdot & & Q_{33}
\end{array}\right]\left[\begin{array}{l}
\left\langle\varepsilon_{11}\right\rangle \\
\left\langle\varepsilon_{22}\right\rangle \\
\left\langle\varepsilon_{12}\right\rangle
\end{array}\right]=\frac{E}{(1+\nu)(1-2 \nu)}\left[\begin{array}{ccc}
1-\nu & \nu & 0 \\
& 1-\nu & 0 \\
\cdot & & 0.5-\nu
\end{array}\right]\left[\begin{array}{l}
\left\langle\varepsilon_{11}\right\rangle \\
\left\langle\varepsilon_{22}\right\rangle \\
\left\langle\varepsilon_{12}\right\rangle
\end{array}\right]
$$

where $Q_{i j}$ are the elements of the resultant elastic constants tensor $\mathbf{Q}, i, j=1,2,3$.

In the considered case, determination of the $\mathbf{Q}$ matrix elements requires performing of 3 independent analyses in the micro scale for each RVE. If the material is linear and fully periodic, only one RVE has to be analysed for the whole structure.

Having determined the components of the $\mathbf{Q}$ matrix, the elastic constants of the equivalent material are calculated according to Eq. (2.5).

\section{Formulation of the optimization problem}

In many engineering optimization problems, more than one optimization criterion have to be taken into account simultaneously. Moreover, the considered criteria are often contradictory, which leads to multiobjective optimization (MOO) tasks. MOO results in a set of trade-off solutions instead of only one optimal solution in single-objective optimization tasks.

The aim of the two-scale multiobjective optimization of composite structures is to find some of its properties in the micro scale (represented by the RVE) which optimize the behaviour of the structure in the macro scale. To solve the boundary value problem in the macro and micro scales, the commercial FEM software MSC Marc and MSC Nastran has been applied. In order to combine MOOPTIM with FEM software, appropriate programming interfaces have been developed. The block diagram of the multiobjective and multiscale evolutionary optimization is presented in Fig. 2.

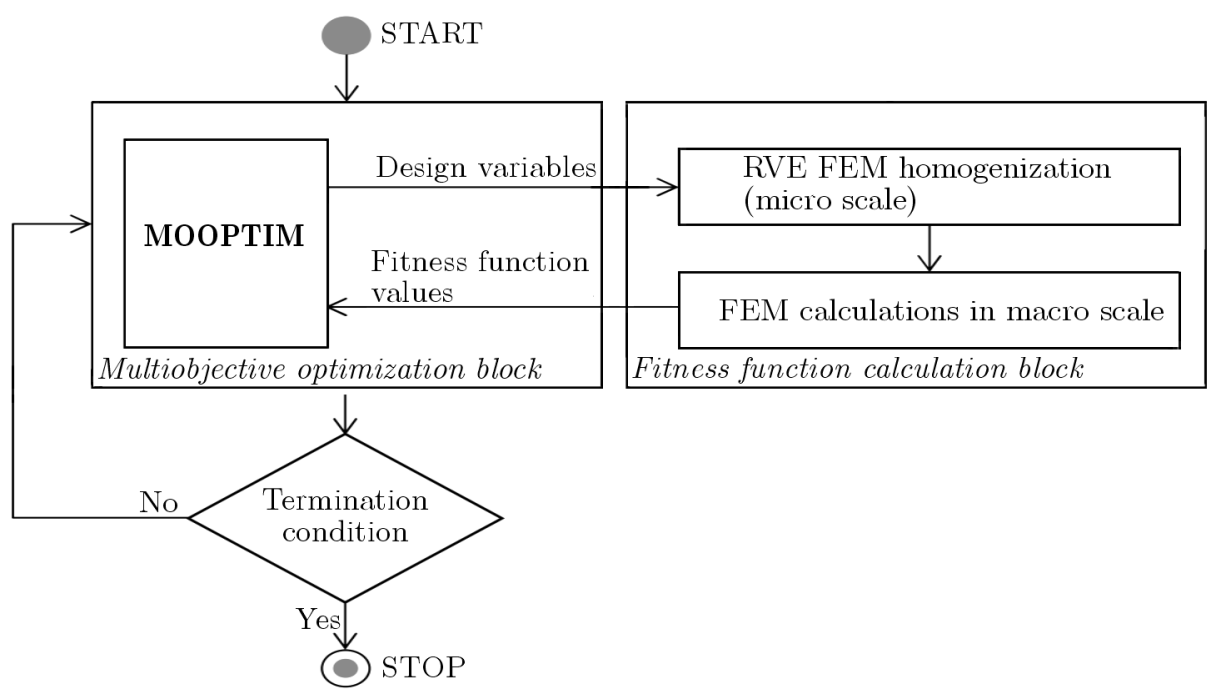

Fig. 2. A block diagram of the multiobjective and multiscale evolutionary optimization 


\subsection{Definition of the multiobjective optimization task}

A MOO problem can be treated as a search for a vector $\mathbf{x} \in \mathbf{D}$, where $\mathbf{D}$ is a set of admissible solutions being a subset of design space $\mathcal{X}$ (Deb, 2001)

$$
\mathbf{x}=\left[x_{1}, x_{2}, \ldots, x_{n}\right]^{\mathrm{T}}
$$

which minimizes the vector of $k$ objective functions

$$
f(\mathbf{x})=\left[f_{1}(\mathbf{x}), f_{2}(\mathbf{x}), \ldots, f_{k}(\mathbf{x})\right]^{\mathrm{T}}
$$

The vector $\mathbf{x}$ has to satisfy $m$ inequality constrains $g_{i}(\mathbf{x}) \geqslant 0, i=1,2, \ldots, m$ and $p$ equality constrains $h_{i}(\mathbf{x})=0, i=1,2, \ldots, p$.

There exist many attitudes to the multi-objective optimization problems (Laumann et al., 2004). A priori methods are based on the transformation of a multiobjective problem into a single-objective one (Collette and Siarry, 2003). The most popular methods from this group are: i) weighted sum method in which each criterion has its own weight value; and ii) $\varepsilon$-constraint method in which the optimization is performed for a chosen criterion while the remaining criteria are treated as constrains. The advantage of the a priori methods is that single-objective methods can be applied, but the drawback is that some very often unrealistic assumptions of the objectives have to be made before the optimization starts.

The second group state interactive methods, which demand an interaction with the decisionmaker (DM) during the optimization to achieve additional information (Luque et al., 2011). There exist many multi-criteria decision-making principles. For example, in Phelps and Koksalan (2003), a pair-wise comparison of solutions is used to include DM's preference. The guided multiobjective evolutionary algorithm (G-MOEA) uses a modified definition of dominance (see: Section 3.2) which has been modified based upon the DM's preference information (Branke and Deb, 2004).

Both aforementioned groups of methods result in one solution of the optimization process. In the a posteriori methods, a set of compromise (trade-off) solutions is determined in the first step of the optimization procedure. The DM is required to choose the most preferred solution in the second step.

\subsection{Pareto concept in multiobjective optimization of composites}

An attitude belonging to a posteriori methods is employed in the present paper. The multiobjective optimization is performed using the Pareto concept of non-dominated solutions (Ehrgott, 2005). If the minimization problem is considered, a solution $\mathbf{x}$ is strongly dominated by the solution $\mathrm{x}^{*}$ if

$$
\forall i \in\{1,2, \ldots, k\}: \quad f_{i}\left(\mathbf{x}^{*}\right)<f_{i}(\mathbf{x})
$$

The solution $\mathbf{x}$ is weakly dominated by the solution $\mathbf{x}^{*}$ if

$$
\begin{array}{ll}
\forall i \in\{1,2, \ldots, k\}: & f_{i}\left(\mathbf{x}^{*}\right) \leqslant f_{i}(\mathbf{x}) \wedge \\
\exists j \in\{1,2, \ldots, k\}: & f_{j}\left(\mathbf{x}^{*}\right)<f_{j}(\mathbf{x})
\end{array}
$$

An example of domination areas for an arbitrary point (solution) $A$ for a two-objective minimization problem is presented in Fig. 3. The set of non-dominated solutions is called the Pareto front.

In the present paper, a multi-objective optimization problem is solved by means of the proposed multiobjective evolutionary algorithm MOOPTIM, which belongs to bio-inspires global optimization methods (see: Section 4). 


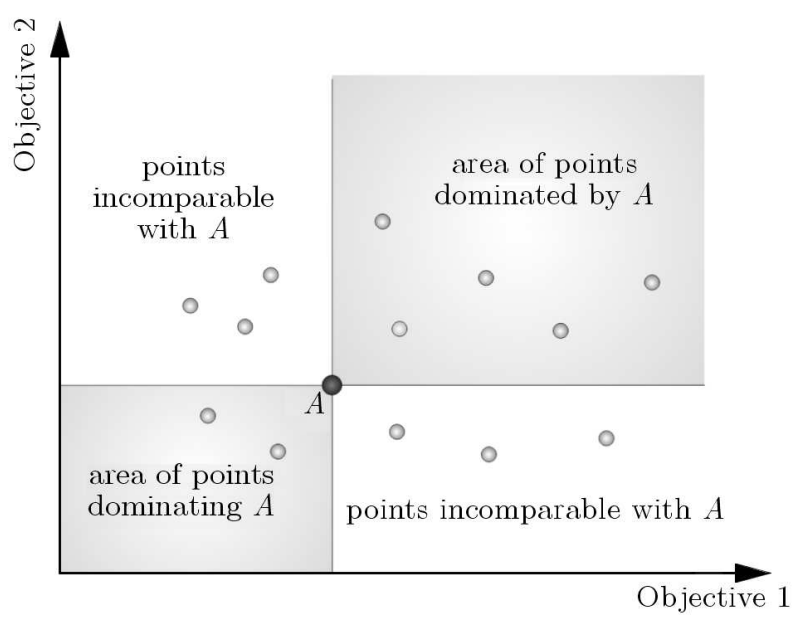

Fig. 3. An exemplary Pareto front and domination areas for point $A$

The real-value coding of the design variables is applied in MOOPTIM. The vector of the design variables (chromosome) has the form

$$
\mathbf{x}=\left[l_{R V E}, E_{m}, E_{f}\right]
$$

where $l_{R V E}$ is the characteristic dimension of the RVE, $E_{m}, E_{f}$ - Young's moduli for the matrix and fibre materials, respectively.

\subsection{Optimization criteria}

The following optimization criteria have been considered simultaneously:

1. The minimization of the (dimensionless) structure $\operatorname{cost} C$

$$
\arg \min \{C(\mathbf{x}) ; \mathbf{x} \in \mathbf{D}\} \quad C(\mathbf{x})=\rho_{f} C_{f} V_{f}(\mathbf{x})+\rho_{m} C_{m} V_{m}(\mathbf{x})
$$

where $\rho_{f}, \rho_{m}$ are fibre and matrix densities, $V_{f}, V_{m}$ - fibre and matrix volumes, $C_{f}, C_{m}$ - fibre and matrix unit costs per kilogram.

2. The minimization of the complementary energy of the structure $\Pi_{\sigma}$ being a measure of the averaged susceptibility of the structure (Burczyński, 1995)

$$
\arg \min \left\{\Pi_{\sigma}(\mathbf{x}) ; \mathbf{x} \in \mathbf{D}\right\} \quad \Pi_{\sigma}(\mathbf{x})=\int_{\Omega} W(\sigma) d \Omega-\int_{\Gamma_{1}} \mathbf{p u}^{0} d \Gamma_{1}
$$

where $W(\sigma)$ is the stress potential related to a volume unit, $\Omega$ - a domain occupied by the body, $\Gamma_{1}$ - part of the boundary on which the function $\mathbf{p u}_{0}$ is defined, $\mathbf{p}, \mathbf{u}_{0}$ - tractions and displacements on $\Gamma_{1}$.

\section{Multiobjective evolutionary algorithm}

An in-house multiobjective evolutionary algorithm MOOPTIM based on the Pareto concept is used for solving optimization tasks (Długosz, 2010). Some ideas in MOOPTIM are inspired by Deb's NSGAII algorithm (Deb et al., 2002). Similarly as in NSGAII, the proposed algorithm uses a non-dominated sorting procedure to classify individuals in the population and a crowding coefficient to preserve diversity in the population. The main differences between MOOPTIM and 
NSGA II are: i) application of a different number and different types of evolutionary operators and ii) selection mechanism.

Two types of mutation (uniform and Gaussian ones) and two crossover operators, in form of a simple crossover and an arithmetical one, are used. As previously tested, the Gaussian mutation operator has significant influence on the effectives of searching ability of the algorithm. This operator requires an extra parameter, called the mutation range, which can take values from 0 to 1 . It is observed that higher values of the mutation range usually improve the convergence of the algorithm, especially for difficult optimization tasks (Długosz, 2013).

Instead of binary tournament selection in NSGA-II, in MOOPTIM, individuals are selected on the basis of a non-domination level as well as the crowding coefficient.

A pseudo code of the algorithm is presented in Fig. 4. In the initialization step, besides determining all settings of the algorithm, the populations $Q_{i}$ and $P_{i}$ of the same size are generated, and the fitness functions are evaluated for the population $Q_{i}$. In the main loop, after the evaluation of the fitness functions values for $P_{i}$, the populations $Q_{i}$ and $P_{i}$ are combined into a set $R_{i}$. Next, the selection procedure is performed on the set $R_{i}$. The individuals from the population $R_{i}$ are selected to $P_{i+1}$ on the basis of the non-domination level and the crowding coefficient. Individuals from $P_{i+1}$ are copied to $Q_{i+1}$ and then, evolutionary operators modify the individuals in the population $P_{i+1}$ to obtain new possible solutions.

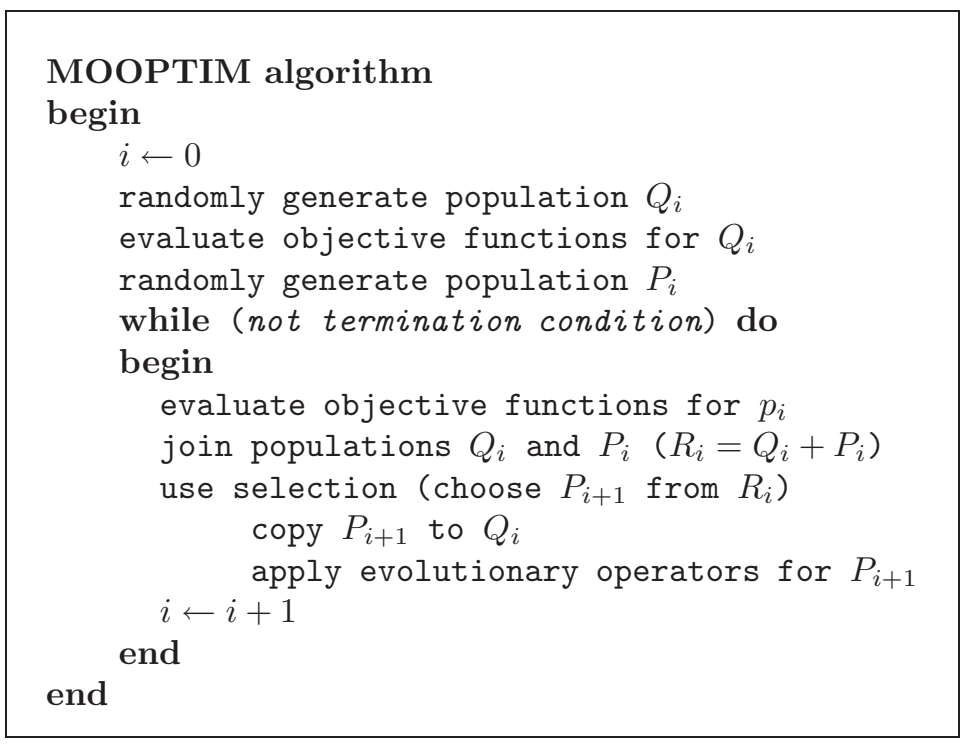

Fig. 4. The pseudo code of the MOOPTIM algorithm

MOOPTIM has been tested on several benchmarks typical for the multiobjective problems like: SCH, ZDT1, ZDT2, ZDT3, ZDT4, ZDT6, CONSTR, SRN, TNK (Deb, 1999; Zitzler and Thiele, 1999). The results obtained using MOOPTIM in most cases are better in comparison with the results obtained by means of NSGA-II. The advantage of using MOOPTIM (instead of NSGA-II) especially appears in the case of functions difficult to optimize, i.e. having strong multimodality, non-convex or a discontinuous Pareto front. Functionals defined for engineering problems, which are solved by using FEM, are usually strongly multimodal and, sometimes, design variables are discontinuous.

The ability of finding global solutions by the optimization algorithm for such problems is essential. The application of the proposed algorithm to solve different optimization tasks has shown its superiority on NSGAII in many cases (Długosz, 2010, 2013). Moreover, MOOPTIM has been successfully applied in the optimization of parameters for porous microstructures in two-scale thermoelastic problems (Długosz, 2014). 


\section{Numerical example}

A box beam structure of dimensions $200 \times 80 \times 60 \mathrm{~mm}$ made of a composite material is considered (Fig. 5). The thickness of each side is constant and equal to $2 \mathrm{~mm}$. The structure is fixed on one end and loaded by a pair of nodal forces of value $P=300 \mathrm{~N}$ each. The structure is divided into 700 Quad4 finite elements having linear shape functions.

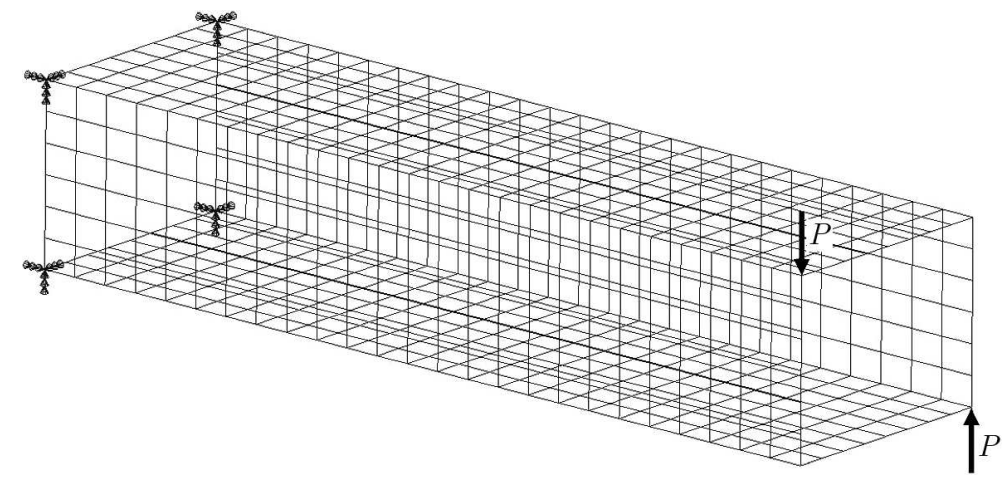

Fig. 5. A box beam - dimensions, mesh and boundary conditions

Global periodicity of the structure is assumed and, as a result, the structure can be fully represented by a RVE containing a single centrally positioned fibre (unit cell) of dimension equal to $15 \mu \mathrm{m}$. The volume fraction of the fibre can vary within the range $4 \%-45 \%$, which is achieved through different sizes of the RVE (RVE side length $l_{R V E}=60-20 \mu \mathrm{m}$ ), as presented in Fig. 6 . Regardless of the fibre volume fraction, each RVE is divided into 820 Quad4 four-node finite elements.

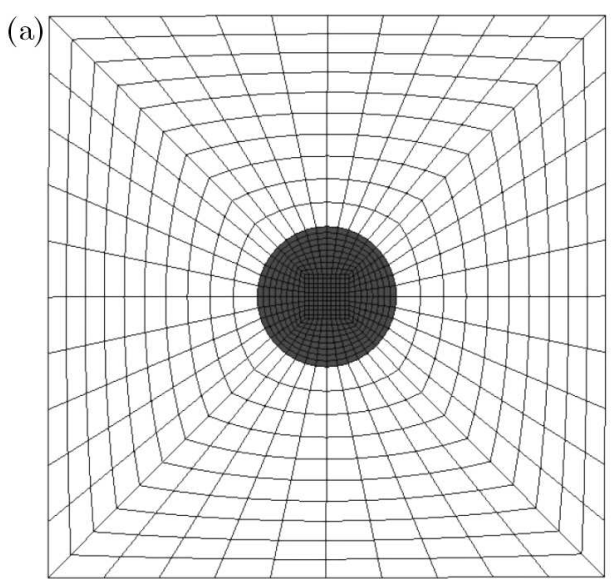

(b)

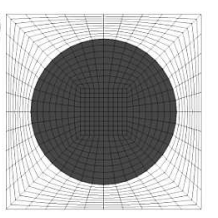

Fig. 6. Exemplary RVEs with mesh for fibre volume fractions: (a) $4 \%$ ( $\mathrm{RVE}_{l}=60 \mu \mathrm{m}$ ), (b) $45 \%\left(\mathrm{RVE}_{l}=20 \mu \mathrm{m}\right)$

The matrix of the composite is an epoxy resin, while different types of fibres may be applied as reinforcement. The fibres are characterized by some parameters, like Young's modulus, Poisson's ratio, density and unit price. The selected parameters of the matrix and fibres materials are collected in Table 1.

It is assumed that the fibre material cost is dependent on material Young's modulus. Two cases are considered:

i) the fibre material cost is approximated by a polynomial function of Young's modulus, as presented in Fig. 7. This attitude assumes the possibility of designing the fibre material that has the desired properties; 
Table 1. Parameters of the composite constituent materials

\begin{tabular}{|c|l|c|c|c|c|}
\hline No. & Material & $\begin{array}{c}E \\
{[\mathrm{GPa}]}\end{array}$ & $\begin{array}{c}\nu \\
{[-]}\end{array}$ & $\begin{array}{c}\text { Density } \\
{\left[\mathrm{g} / \mathrm{cm}^{3}\right]}\end{array}$ & $\begin{array}{c}\text { Unit price } \\
{[\in / \mathrm{kg}]}\end{array}$ \\
\hline \hline 1 & E glass (fibre) & 72 & 0.22 & 2.54 & 1.5 \\
\hline 2 & S-2 glass (fibre) & 87 & 0.22 & 2.49 & 5 \\
\hline 3 & HS carbon (fibre) & 230 & 0.2 & 1.8 & 25 \\
\hline 4 & IM carbon (fibre) & 285 & 0.2 & 1.8 & 55 \\
\hline 5 & HM carbon (fibre) & 400 & 0.2 & 1.8 & 175 \\
\hline 6 & Epoxy resin (matrix) & 2.4 & 0.35 & 1.14 & 8 \\
\hline
\end{tabular}

ii) the fibre material cost is taken from the database of material parameters (materials 1-5 from Table 1.

Each chromosome which is a design variable vector consists of two genes representing:

i) size of the RVE and Young's modulus of the fibre (case i);

ii) size of the RVE and the fibre material number (case ii).

Two variants of the case i) are taken into consideration, as presented in Fig. 7:

i) only carbon fibres are considered (solid line);

ii) carbon and glass fibres are taken into account (dashed line).

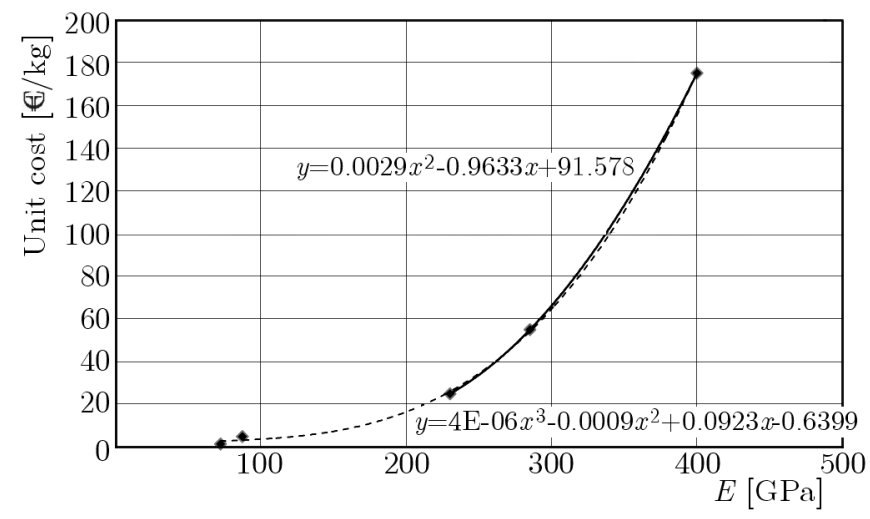

Fig. 7. Young's modulus of the fibre - unit cost diagrams for polynomial approximation

The results of the optimization in form of Pareto frontiers for cases i) and ii) are presented in Fig. 8.

As revealed from Fig. 8, the best optimization results have been obtained for a wider search space (glass and carbon fibres).

The design variables values, fibre volume fractions and fitness function values for exemplary points 1-4 for carbon and glass fibres with different approximations are collected in Table 2.

The following parameters of MOOPTIM are assumed:

- probability of the Gaussian mutation $p_{g m}=0.7$;

- probability of the uniform mutation $p_{u m}=0.1$;

- probability of the simple crossover $p_{s c}=0.1$;

- probability of the arithmetic crossover $p_{a c}=0.1$;

- number of chromosomes $n_{c h}=70$;

- number of generations $n_{g}=70$. 


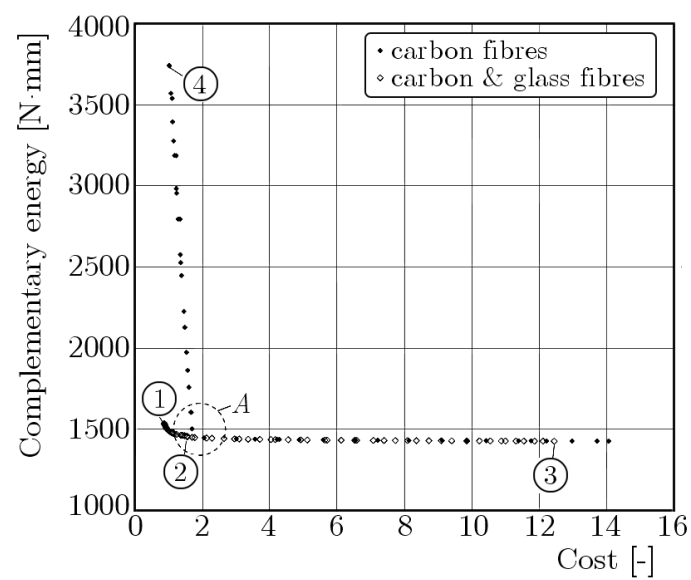

Fig. 8. Box beam - results of multiobjective optimization for case i)

Table 2. Multiobjective optimization results for the box beam

\begin{tabular}{|c|c|c|c|c|}
\hline \multirow{2}{*}{ Point } & \multicolumn{2}{|c|}{ Design variable } & \multicolumn{2}{c|}{ Fitness function } \\
& $\begin{array}{c}\text { RVE length }[\mu \mathrm{m}] / \text { fibre } \\
\text { volume fraction }[\%]\end{array}$ & $\begin{array}{c}\text { E of the fibre } \\
{[\mathrm{GPa}]}\end{array}$ & $\begin{array}{c}\text { Structure unit } \\
\text { cost }[-]\end{array}$ & $\begin{array}{c}\text { Complementary } \\
\text { energy }[\mathrm{Nm}]\end{array}$ \\
\hline \hline 1 & $20 / 45$ & 70 & 0.848 & 1541.43 \\
\hline 2 & $20 / 45$ & 204.6 & 1.754 & 1454.38 \\
\hline 3 & $20 / 45$ & 400 & 12.429 & 1431.94 \\
\hline 4 & $58.0764 / 5.24$ & 70 & 0.941 & 3736.95 \\
\hline
\end{tabular}

The multiobjective optimization results for case ii) (the choice of the material from the database) compared to the results obtained for case i) (carbon and glass fibres, polynomial approximation, also shown in Fig. 8), are presented in Fig. 9.

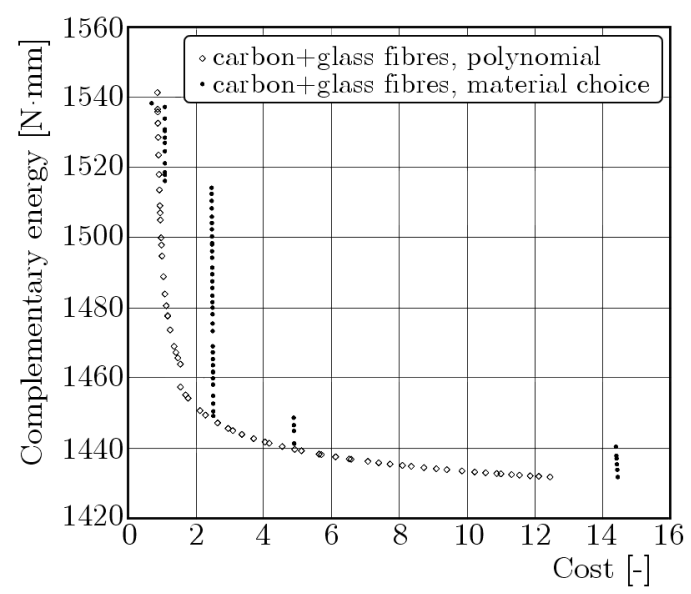

Fig. 9. Box beam - results of multiobjective optimization for cases i) and ii)

The information obtained from the results for the approximated cost values may be used to search for or for designing materials the cost of which is a function of selected parameters. The multiobjective optimization in the case where materials are chosen from the database of the available materials is more practical, but typically gives worse optimization results due to narrower search space. 


\section{Final conclusions}

The multiscale and multiobjective optimization of heterogeneous structures has been performed. Periodic fibre-reinforced composite structures have been examined. The RVE concept has been employed to perform numerical homogenization. FEM calculations have been performed to solve boundary-value problems in both scales. Two contradictory optimization criteria have been considered to obtain the optimal behaviour of the macrostructure modifying the volume fraction of the fibre and the fibre material in the micro scale. An in-house multiobjective evolutionary algorithm MOOPTIM has been applied to solve the multiobjective optimization problems.

The proposed approach enables designing of composite microstructure based on the criteria defined in the macro scale. The paper presents an example with a unchangeable (circular) shape of the reinforcement. In that case, the optimization was to determine the reinforcement material and its volume fraction in the RVE.

The optimization results have been presented in form of Pareto frontiers of non-dominated solutions and in graphical form of optimal microstructures for selected non-dominated solutions. Graphical presentation of the non-dominated solutions also carries information about the nature of the conflict between the criteria. For instance, the frontier presented in Fig. 8 for variant i) (carbon fibres) has two distinct areas merging at an approximately straight angle, while the front for variant ii) (carbon and glass fibres) does not have clearly demarcated sub-areas. These places at the Pareto front, where a small change in the value of one of the objective function results in a large change in the value of other objective functions (area $A$ in Fig. 8) require particular attention in the design process.

The application of the MOOPTIM algorithm to the considered multiobjective problems proves that the proposed algorithm is useful. The defining and using of the optimization criteria other than those presented in this paper do not pose problems in the proposed approach.

\section{References}

1. Beluch W., Burczyński T., 2014, Two-scale identification of composites' material constants by means of computational intelligence methods, Archives of Civil and Mechanical Engineering, 14, 4, 636-646

2. Beluch W., Burczyński T., DŁugosz A., 2008, Evolutionary multi-objective optimization of laminates, [In:] Evolutionary Computation and Global Optimization, J. Arabas (Edit.), Warsaw University of Technology Publishing House, Scientific Papers, Electronics 165, 43-50

3. Bensoussan A., Lionis J.L., Papanicolaou G., 1978, Asymptotic Analysis for Periodic Structures, North-Holland, Amsterdam

4. Branke J., Deb K., 2004, Integrating user preferences into evolutionary multi-objective optimization, [In:] Knowledge Incorporation in Evolutionary Computation, Y. Jin (Edit.), Springer, 461-477

5. Brebbia C.A., Dominiguez J., 1989, Boundary Elements an Introductory Course, Computational Mechanics Publications, Southampton Boston

6. Buryachenko V., 2007, Micromechanics of Heterogeneous Materials, Springer, New York

7. Burczyński T., 1995, Boundary Element Method in Mechanics (in Polish), WNT, Warsaw

8. Burczyński T., Mrozek A., Kuś W., 2007, A computational continuum-discrete model of materials, Bulletin of the Polish Academy of Sciences - Technical Sciences, 55, 1, 85-89

9. Collette Y., Siarry P., 2003, Multiobjective Optimization: Principles and Case Studies, Springer 
10. De Castro L.N., Timmis J., 2002, Artificial Immune Systems: a New Computational Intelligence Approach, Springer

11. DeB K., 1999, Multi-objective genetic algorithms: problem difficulties and construction of test problems, Evolutionary Computation, 7, 3, 205-230

12. Deb K., 2001, Multi-Objective Optimization Using Evolutionary Algorithms, Wiley

13. Deb K., Pratap A., Agarwal S., Meyarivan T., 2002, A fast and elitist multi-objective genetic algorithm: NSGA-II, IEEE Transactions on Evolutionary Computation, 6, 2, 181-197

14. DŁugosz A., 2010, Multiobjective evolutionary optimization of MEMS structure, Computer Assisted Mechanics and Engineering Sciences, 17, 1, 41-50

15. DŁugosz A., 2013, Multicriteria Optimization in Coupled Field Problems (in Polish), Monography (497), Silesian University of Technology Publishing House

16. DŁugosz A., 2014, Optimization in multiscale thermoelastic problems, Computer Methods in Materials Science, 14, 1, 86-93

17. Ehrgotт M., 2005, Multicriteria Optimization, Springer

18. Eshelby J.D., 1957, The determination of the field of an ellipsoidal inclusion and related problems, Proceedings of the Royal Society of London, 241, 376-396

19. Gibson R. F., 2012, Principles of Composite Material Mechanics, CRC Press

20. Hashin Z., 1964, Theory of mechanical behavior of heterogeneous media, Applied Mechanics Reviews, 17, 1-9

21. Hill R., 1963, Elastic properties of reinforced solids: Some theoretical principles, Journal of the Mechanics and Physics of Solids, 11, 357-372

22. Ilic S., HackL K., 2009, Application of the multiscale FEM to the modeling of nonlinear multiphase materials, Journal of Theoretical and Appllied Mechanics, 47, 3, 537-551

23. Kennedy J., Eberhart R., 2001, Swarm Intelligence, Morgan Kaufmann, San Francisco

24. Kouznetsova V., 2002, Computational homogenization for the multi-scale analysis of multi-phase materials, Ph.D. thesis, Technische Universiteit Eindhoven

25. Kröner E., 1972, Statistical Continuum Mechanics, Springer, Berlin

26. KuCzma M., 2014, Two-scale numerical homogenization of the constitutive parameters of reactive powder concrete, International Journal for Multiscale Computational Engineering, 2, 5, 361-374

27. Laumann M., Zitzler E., Bleuler S., 2004, A tutorial on evolutionary multiobjective optimization, Metaheuristics for multiobjective optimisation, [In:] Lecture Notes in Economics and Mathematical Systems, X. Gandibleux, M. Sevaux, K. Sorensen, V. T'kindt (Edit.), Springer, 3-37

28. Luque M., Ruiz F., Miettinen K., 2011, Global formulation for interactive multiobjective optimization, OR Spectrum, 33, 1, 27-48

29. Madi K., Forest S., Jeulin D., Boussuge M., 2006, Estimating RVE Sizes for 2D/3D Viscoplastic Composite Materials, HAL - CCSD

30. Michalewicz Z., Fogel D., 2004, How to Solve It: Modern Heuristics, Springer Science \& Business Media

31. Nemat-Nasser S., Hori M., 1993, Micromechanics: Overall Properties of Heterogeneous Materials, Elsevier, Amsterdam

32. Phelps J.S., Koksalan M., 2003, An interactive evolutionary metaheuristic for multiobjective combinatorial optimization., Management Science, 49, 12, 1726-1738

33. Ptaszny J., Fedeliński P., 2011, Numerical homogenization by using the fast multipole boundary element method, Archives of Civil and Mechanical Engineering, 11, 1, 181-193 
34. TAKAno N., ZAKo M., 2000, Integrated design of graded microstructures of heterogeneous materials, Archive of Applied Mechanics, 70, 8/9, 585-596

35. Vernerey F.J., KABIRI M., 2014, Adaptive concurrent multiscale model for fracture and crack propagation in heterogeneous media, Computer Methods in Applied Mechanics and Engineering, 276, 566-588

36. Zhoua A., Qu B., Li H., Zhao S., Suganthan P., Zhang Q., 2011, Multiobjective evolutionary algorithms: a survey of the state of the art, Swarm and Evolutionary Computation, 1, 32-49

37. Zienkiewicz O.C., Taylor R.L., 2000, The Finite Element Method, vol. 1-3, Butterworth, Oxford

38. Zitzler E., Thiele L., 1999, Multiobjective evolutionary algorithms: a comparative case study and the strength Pareto approach, IEEE Transactions on Evolutionary Computation, 3, 4, 257-271

39. Zohdi T.I., Wriggers P., 2005, An introduction to computational micromechanics, Lecture Notes in Applied and Computational Mechanics, Springer-Verlag

Manuscript received May 7, 2015; accepted for print August 6, 2015 